\title{
Estado de la investigación sobre la comprensión de la lectura en estudiantes universitarios
}

\author{
Magda Yuliza Quintero-Ramírez * \\ Yeraldín Vela-Valderrama**
}

Estudiante de noveno semestre,

Licenciatura en Lengua Castellana y

Literatura, Universidad de la Amazonia,

Florencia, Caquetá.

Correo electrónico

yulizaquinteror@hotmail.com

** Estudiante de noveno semestre,

Licenciatura en Lengua Castellana y

Literatura, Universidad de la Amazonia,

Florencia, Caquetá.

Correo electrónico:

geral.02vela@gmail.com

Recibido: 25 de enero del 2016

Aprobado: 25 de febrero del 2016

Cómo citar este artículo: Quintero-Ramírez, Magda y Yeraldín Vela-Valderrama. "Estado de la investigación sobre la comprensión de la lectura en estudiantes universitarios". Rastros Rostros 18.32 (2016): 51-65. Impreso. doi: http://dx.doi.org/10.16925/ra.v18i32.1177.

\section{Resumen}

Introducción: el presente artículo analiza las investigaciones de los últimos cinco años sobre la comprensión lectora de estudiantes universitarios (20092014). Estas debían tener temáticas recientes, innovadoras, relevantes y estar basadas en la identificación de herramientas, estrategias, dificultades y aspectos menos evidentes que contribuyeran al desarrollo de un nuevo conocimiento para ser utilizado como instrumento en la recolección de otros análisis. Se encontraron temas como la compresión de textos, sus procesos y sus características. Todo esto se realizó mediante la línea de investigación de didáctica de la lengua castellana y la literatura de la Universidad de la Amazonia y aprobada por el Comité de Investigaciones del programa de Lengua Castellana y Literatura de la Facultad de Ciencias de la Educación. Metodología: se analiza el contenido de 50 investigaciones en los ejes de metodología, referentes teóricos, tendencias, con el fin de obtener un balance a nivel nacional e internacional. Resultados: se identifican falencias, técnicas y elementos en torno a los procesos de lectura y comprensión en estudiantes universitarios. Conclusiones: la investigación permite implantar una visión general de las condiciones actuales que dominan en el campo de lectura y determina la falta de noción acerca de las perspectivas lectoras que logran desarrollar los estudiantes en esta constante tarea.

Palabras clave: comprensión de lectura, estudiante de la universidad, educación, formación, lectura. 


\title{
The State of Research on Reading Comprehension in University Students
}

\begin{abstract}
Introduction: This article analyzes research from the past five years on the reading comprehension of university students (2009-2014). This research had to contain topics that were recent, innovative, relevant, and based on the identification of tools, strategies, difficulties, and less obvious aspects that contribute to the development of new knowledge to be used as a tool in the collection of other analyses. Topics such as text comprehension and its processes and characteristics were among those found. All of this was carried out through the line of research of teaching of the Spanish language and literature at the Universidad de la Amazonia and approved by the Research Committee of the Spanish Language and Literature Program of the Department of Education Sciences. Methodology: The content of 50 research studies with a focus on methodology, theoretical referents, and trends was analyzed in order to obtain an assessment at the national and international level. Results: Deficiencies, techniques, and elements related to the processes of reading and understanding in university students were identified. Conclusions: The research serves as an overview of the current conditions that dominate in the field of reading and indicates a lack of awareness of the reader expectations that students manage to develop in this constant task.
\end{abstract}

Keywords: reading comprehension, university student, education, training, reading.

\section{Estado da pesquisa sobre a compreensão da leitura em estudantes universitários}

\section{Resumo}

Introdução: o presente artigo analisa as pesquisas dos últimos cinco anos sobre a compreensão leitora de estudantes universitários (2009-2014). Estas deviam ter temáticas recentes, inovadoras, relevantes e estar baseadas na identificação de ferramentas, estratégias, dificuldades e aspectos menos evidentes que contribuíssem para o desenvolvimento de um novo conhecimento para ser utilizado como instrumento na coleta de outras análises. Encontraram-se temas como a compreensão de textos, seus processos e suas características. Tudo isso se realizou mediante a linha de pesquisa didática da língua castelhana e da literatura da Universidad de la Amazonia e aprovada pelo Comitê de Pesquisas do Programa de Língua Castelhana e Literatura da Faculdade de Ciências da Educação. Metodologia: analisa-se o conteúdo de 50 pesquisas nos eixos de metodologia, referentes teóricos, tendências, com o objetivo de obter uma avaliação no âmbito nacional e internacional. Resultados: identificam-se falências, técnicas e elementos em torno dos processos de leitura e compreensão em estudantes universitários. Conclusões: a pesquisa permite implantar uma visão geral das condições atuais que dominam no campo de leitura e determina a falta de noção acerca das perspectivas leitoras que os estudantes conseguem desenvolver nessa constante tarefa.

Palavras-chave: compreensão de leitura, estudante da universidade, educação, formação, leitura. 


\section{Introducción}

La lectura es imprescindible en el ser humano; de hecho, gracias a esta se puede conocer la cultura, la información y el conocimiento que existe en la sociedad y tener acceso a estos fenómenos. Según Llanos, "[...] la comprensión de textos es la base del desarrollo de todo individuo" (15). Cabe decir que la lectura es un objetivo y un medio para el aprendizaje, y favorece el proceso de aprender. De esta forma, se puede utilizar la lectura como una herramienta eficaz para adquirir saberes y transformar el conocimiento, pues, para Rincón y Gil, “[...] el funcionamiento de la vida democrática y de la vida social, hoy en general, está mediado por la lectura y la escritura" (1).

Desde que tenemos conocimiento estamos expuestos a interpretar y analizar textos de todo tipo, dado que la importancia de leer no se debe solo al contenido, sino también a la cantidad, el estilo y la intención de cada lectura. Del mismo modo, en las aulas universitarias es esencial la comprensión de documentos científicos. Lastimosamente, muchos de los estudiantes que ingresan a la universidad carecen de este tipo de destrezas, lo cual hace muy difícil el proceso de enseñanza-aprendizaje. Para superar esta carencia debe trabajarse arduamente durante la vida académica.

Este trabajo empieza cuando se identifican las problemáticas relacionadas con la enseñanza y aprendizaje de los contenidos de la lengua por medio de la interacción entre el estudiante universitario y el texto. Este tipo de investigación es de gran importancia, pues gracias a esta es posible identificar el origen de estos problemas y plantear un plan de acción para mitigar las falencias en torno a la comprensión de textos en el nivel universitario.

Al comienzo se desconocía el desarrollo de aportes teóricos y metodológicos de las investigaciones sobre la comprensión de textos en jóvenes universitarios, por lo cual se plantea la pregunta: ¿cuál es el estado del arte de las investigaciones acerca de la comprensión de lectura en universitarios?

Resolver este interrogante es importante para conocer el estado del arte en las investigaciones sobre la comprensión de lectura en los estudiantes universitarios y las tendencias investigativas efectuadas durante los últimos cinco años, de manera que se puedan solucionar las falencias observadas y contribuir a la creación de nuevas estrategias de enseñanza. También puede servir como guía investigativa para futuros trabajos investigativos.
El objetivo de este trabajo es identificar las características de las investigaciones acerca de la comprensión de textos en estudiantes universitarios teniendo en cuenta la línea de investigación de didáctica de la lengua castellana y la literatura.

Este trabajo es un artículo de revisión documental en el que se buscaron textos realizados durante los últimos cinco años sobre la comprensión de lectura en universitarios, luego se organizó esta información en una base de datos de Microsoft Access. Para esto se realizó un análisis hermenéutico y se examinaron investigaciones internacionales y nacionales, además se destacaron los elementos del tema objeto de investigación.

\section{Metodología}

Este estudio es una investigación de tipo documental. Vélez afirma que este tipo de investigación tiene como objetivo:

[...] el desarrollo de las capacidades reflexivas y críticas a través del análisis, interpretación y confrontación de la información regida. Entre los posibles propósitos de este tipo de investigación se encuentran: describir, mostrar, probar, persuadir o recomendar. La investigación debe llevar a resultados originales y de interés para el grupo social de la investigación.

Se obtuvo información de 50 investigaciones acerca de la comprensión lectora en estudiantes universitarios para obtener y analizar de forma detallada los resultados y la importancia que fundamenta este tema.

Este proceso tiene un enfoque cuantitativo y cualitativo. Se recolectó la información, se cuantificaron y analizaron los datos; además, se hizo una interpretación basada en el tipo de documento para clasificar y organizar su estructura, sin perder de vista el tema central.

Por otra parte, para la recopilación de los documentos se tuvieron en cuenta que fueran cincuenta trabajos de investigación; que el tiempo máximo de publicación no fuera mayor a cinco años (publicados aproximadamente entre el 2009 y el 2014), y que fueran artículos científicos, tesis de maestría o doctorado, y ponencias.

La investigación tuvo dos fases: una heurística y una hermenéutica. En la primera fase, se buscó información en las bases de datos ProQuest, Google Académico, Scielo, Redalyc. Esta fase definió la comprensión de lectura en universitarios como campo 
de estudio. Todos los datos consultados fueron organizados en una base de datos realizada en Microsoft Access. Alguna de esta información se recopiló y organizó en diferentes categorías: nombre del autor, título, año, país, objetivo, problema, fundamentos teóricos, metodológicos, técnicas e instrumentos, y resultados.

En la segunda fase, se analizó el contenido, se tuvo en cuenta la ubicación general y específica de cada investigación, si era internacional o nacional. Asimismo, se hizo un análisis detallado para tener en consideración los problemas, enfoques teóricos y metodológicos, resultados y tendencias, de manera que se obtuvieran algunas conclusiones.

\section{Resultados}

Los resultados de estudio se presentan en orden descendente en relación con la cantidad de investigaciones realizadas por países durante los últimos años en función de la comprensión de lectura en estudiantes universitarios.

\section{Ámbito internacional}

Los países que son eje central en estas investigaciones son de América Central y del Sur. México y Colombia han sido los principales protagonistas en estas indagaciones, mientras Costa Rica, Uruguay y Cuba tienen una participación minoritaria. En el continente europeo, España posee un desarrollo investigativo menor al de países sobresalientes en las pesquisas acerca de la comprensión de lectura en estudiantes universitarios:

$\mathrm{Al}$ analizar las 50 investigaciones internacionales acerca de la comprensión lectora en universitarios, se ve, en términos cuantitativos, que México y Colombia son los países con mayor presencia en el ámbito investigativo. En estos países se examina la comprensión textual en las aulas universitarias y tienen los mayores porcentajes de las investigaciones realizadas sobre el tema. Las investigaciones constituyen más del $50 \%$ como se observa en la figura $1, y$ superan en gran medida a los estudios que se hacen en otros países latinoamericanos y en España.

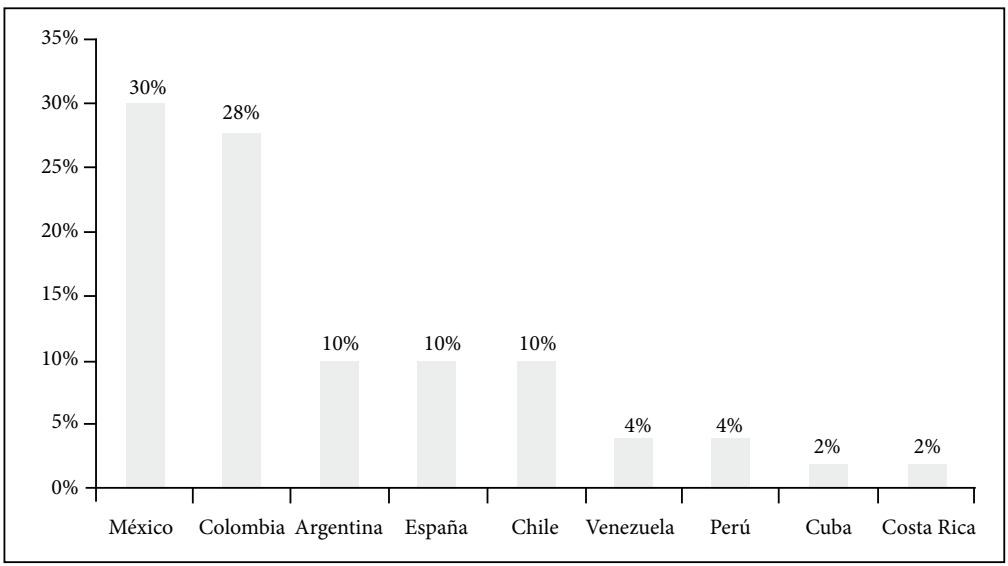

Figura 1. Porcentaje de las investigaciones en el ámbito internacional. Fuente: elaboración propia.

México: de acuerdo con lo anterior, en la tabla 1 se presenta a México como el que tiene mayores investigaciones con 15.

Principalmente, se presenta el interés por conocer y analizar la importancia que tiene la comprensión de lectura, como lo mencionan Monroy y Gómez: "Hoy en día se habla de la necesidad de que las nuevas generaciones aprendan a desarrollar habilidades, estrategias cognitivas y metacognitivas que les permitan concretar aprendizajes" (1).

A propósito de esto, Fernández asegura:

Si bien, la lectura ha mantenido su esencia como medio de comunicación, las concepciones sociales y pedagógicas que hay en torno a este proceso han ido variando a lo largo del tiempo en función de las necesidades particulares del contexto. (15) 
Tabla 1. Investigaciones sobre la comprensión de lectura en México

\begin{tabular}{|l|l|}
\hline Año & \multicolumn{1}{c|}{ Investigaciones } \\
\hline 2009 & $\begin{array}{l}\text { Monroy-Romero y Gómez-López } \\
\text { Hernández-Suárez y Riffo } \\
\text { González-Hernández }\end{array}$ \\
\hline 2010 & $\begin{array}{l}\text { Morales, Cruz, León, Silva, Arroyo, y Carpio } \\
\text { Alfaro-López }\end{array}$ \\
\hline 2011 & Moore-Hanna y Narciso-Sánchez \\
\hline 2013 & $\begin{array}{l}\text { Fernández de Castro } \\
\text { Guevara-Benítez y Guerra-García } \\
\text { Cepeda-Islas, López-Gamiño y Santoyo-Velasco } \\
\text { Guerra-García y Guevara-Benítez } \\
\text { Mendes-Pessoa, Moraes-Goes, y Kito } \\
\text { Vega-López, Báñales-Faz y Reyna Valladares } \\
\text { Castro-Azuara y Sánchez-Camargo }\end{array}$ \\
\hline 2014 & \begin{tabular}{l} 
Aguilar-Trejo, Ramírez Martinell y López González \\
\hline
\end{tabular} \\
\hline
\end{tabular}

Fuente: elaboración propia.

A partir de la cita anterior se observa que la lectura en la educación universitaria es un factor importante en la vida de todas las personas y, en especial, la de los jóvenes, pues esta se convierte en la base fundamental de su formación y les permite entrar en una lucha constante por estimular sus destrezas. Sin embargo, son pocos los que adquieren un hábito lector en su formación.

Igualmente Guerra y Guevara afirman que “[...] los problemas de comprensión lectora en estudiantes universitarios residen principalmente en la identificación de la o las ideas principales" (2). Por esta razón, proponen que el profesor genere herramientas que le permita conocer el nivel de comprensión lectora de cada uno de sus alumnos sin invertir un tiempo excesivo. Aguilar, Ramírez y López afirman que "[...] el conocimiento y la información juegan un papel fundamental para activar las interacciones entre las personas" (2).

De esta forma, de acuerdo con Hernández y Riffo, "[...] se requiere no solo conocer los hábitos de lectura de los universitarios, sino describir el procesamiento de lectura seguido por ellos cuando enfrentan textos académicos" (3). Todo esto se hace con el fin de identificar realmente sus puntos fuertes y débiles, lo que ayuda a desarrollar una mejor estrategia para lograr que comprendan e interpreten los textos y así elevar el desarrollo argumentativo de los estudiantes.

Para Morales, Cruz, León, Silva, Arroyo y Carpio, "[...] el hacer del estudiante cuando lee es un referente empírico de los procesos mentales” (2). La lectura permite crear diferentes mundos, que se forman con cada conocimiento logrado a partir de cada texto leído. Igualmente, "[...] se recomiendan el tipo de estrategias como el subrayar, el uso de marca-textos, el uso de marcas o números" (Cepeda, López y Santoyo 7) para lograr el mejoramiento del proceso lector con la implementación de diferentes métodos.

Cabe resaltar que "[...] la mayoría de los lectores se limitan a comentar su lectura de los textos impresos que tradicionalmente se consideran lectura" (Moore y Sánchez 12). Un factor esencial es reconocer cuáles son los textos de relevancia y los que aportan al desarrollo propio y social. Además, "[...] leer implica poner en marcha el esquema mental para proyectarlo sobre el texto" (Alfaro 6): leer es algo individual y particular que implica liberar más el pensamiento.

En esta perspectiva, "[...] los profesores no solo deben enseñar a los alumnos a leer los diferentes tipos de texto indicados en el curso, sino que analicen cómo ellos lo hacen y los inciten a ser más autónomos cuando leen" (Mendes, Moraes y Kito 14). De esta forma, se puede fomentar el desarrollo de la autonomía mental de los alumnos, lo que indica que existe una gran responsabilidad en el trabajo docente.

Por otra parte, "[...] la comprensión de múltiples documentos es uno de los principales retos del aprendizaje disciplinar al que se enfrentan los estudiantes universitarios enfatizan" (Vega, Bañales y Reyna 19), pues, como se sabe, el hecho de leer un texto no implica que el estudiante lo comprenda y, por consiguiente, “[...] la búsqueda de información y la lectura de textos especializados es la base para desarrollar las habilidades argumentativas requeridas en la universidad" (Castro y Sánchez 22). Así, se debe fomentar el hecho de que los estudiantes sean autodidactas y no se limiten a leer única y exclusivamente los textos dirigidos y exigidos por el profesor, ya que al tener leído el material extra se garantiza una mayor comprensión y un material argumentativo de calidad. Esto a ayuda a que las clases sean más fructíferas y tengan interés, lo que deja en el pasado la escuela tradicional en la cual solo el profesor era el poseedor del conocimiento y solo él era el encargado de transmitirlo a sus estudiantes sin objeción alguna. Más adelante se podrá observar a Colombia, que cuenta con un $28 \%$ de las investigaciones. Este análisis estará centrado en el ámbito nacional para ver más a fondo las problemáticas existentes en el país y porque da una orientación para futuras investigaciones dentro de este.

Argentina: a continuación, se muestran las investigaciones de Argentina, en las cuales se destaca el interés 
por identificar las posibles causas de las dificultades sobre comprensión textual (tabla 2). Seguidamente se podrá observar los datos de estas investigaciones y sus autores.

Tabla 2. Investigaciones sobre la comprensión de lectura en Argentina

\begin{tabular}{|l|l|c|}
\hline \multicolumn{1}{|c|}{ Autores } & \multicolumn{1}{|c|}{ Título } & Año \\
\hline Heit & $\begin{array}{l}\text { Estrategias metacognitivas de } \\
\text { comprensión lectora y eficacia en la } \\
\text { asignatura Lengua y Literatura }\end{array}$ & 2012 \\
\hline $\begin{array}{l}\text { Saux, Burin, Irraza- } \\
\text { bal y Molinari-Ma- } \\
\text { rotto }\end{array}$ & $\begin{array}{l}\text { Inferencias causales durante la } \\
\text { comprensión de textos expositivos } \\
\text { en formato multimedia }\end{array}$ & 2012 \\
\hline $\begin{array}{l}\text { Demagistri y } \\
\text { Naveira }\end{array}$ & $\begin{array}{l}\text { Comprensión de textos científicos en } \\
\text { adolescentes }\end{array}$ & 2010 \\
\hline $\begin{array}{l}\text { Cartoceti, Abu- } \\
\text { samra, Sampedro y } \\
\text { Ferreres }\end{array}$ & $\begin{array}{l}\text { Influencia del mecanismo de actua- } \\
\text { lización en la comprensión de textos }\end{array}$ & 2010 \\
\hline Dorronzoro & $\begin{array}{l}\text { Las prácticas de lectura y escritura } \\
\text { en el contexto de la educación } \\
\text { superior: Una aproximación socio- } \\
\text { cultural }\end{array}$ & 2013 \\
\hline
\end{tabular}

Fuente: elaboración propia.

Según lo expuesto en la tabla 2, Argentina es uno de los países con mayor número de investigaciones: hay cinco trabajos. Cuatro de estos corresponden a artículos científicos y solo uno a una tesis de pregrado.

Se resaltan algunos aportes como el de Heit (17) respecto a la utilización de estrategias globales (conocer el propósito de la lectura, anticipar el contenido del texto, usar las claves contextuales, entre otras) para favorecer un aprendizaje más autónomo y con un mayor rendimiento. Saux, Irrazabal, y Molinari mencionan que "[...] tareas de elevada dificultad intrínseca acarrean un aumento de la carga cognitiva" (3). Sobre esto Demagistri y Naveira afirman que "[...] su utilización permite optimizar los procesos de enseñanza aprendizaje dando lugar al desarrollo de estrategias de intervención psicoeducativas" (6).

Se necesita leer una variedad de textos para apropiarse de diferentes conocimientos y la importancia del hecho no solo radica en los contenidos, sino en la cantidad, estilo y propósitos de la lectura (Salas). Estos factores son los encargados de generar un buen proceso de comprensión durante los niveles de educación.

Según Cartoceti, Abusamra, Sampedro, y Ferrere, "[l]a comprensión de textos es una tarea cognitiva compleja que requiere de la integridad de múltiples procesos y habilidades" (2). Por este motivo es posible comprender que, para que se comprenda un texto de forma correcta sin dejar de lado el libre pensamiento, es necesaria la interacción e integración entre profesor-alumno y de todos los entes del ambiente educativo:

Se han constituido como objetos de estudio a partir de interrogantes planteados desde el marco sociocultural, vinculados con el para qué se lee, el qué se lee y el cómo se lee en la universidad, y abordados desde tres perspectivas de estudio que entendemos complementarias con el marco original: la perspectiva de las representaciones sociales, la perspectiva del interaccionismo socio-discursivo y la de la psicología del desarrollo. (Dorronzoro 98)

España: se presentan las investigaciones españolas, en las cuales se resalta el estudio de las dificultades de los jóvenes universitarios y las orientaciones para que estos comprendan los textos de forma eficaz (tabla 3).

En España, las investigaciones orientan y hablan acerca de los procesos de comprensión lectora de textos así como de estrategias metacognitivas e interactivas. Según Casanny “[...] leer también es algo que está muy relacionado con la construcción de identidades, con la asunción de los roles sociales de lector y de autor" (6). Sobre esto afirma Bustos: "[1]a lectura comprensiva es un proceso muy complejo, donde el lector llega a extraer el significado que subyace en un texto, relacionándolo con sus experiencias previas" (1).

Tabla 3. Investigaciones sobre la comprensión de lectura en España

\begin{tabular}{|l|l|l|}
\hline \multicolumn{1}{|c|}{ Autores } & \multicolumn{1}{|c|}{ Título } & AÑO \\
\hline Bustos-Pérez & $\begin{array}{l}\text { Dificultades de la comprensión } \\
\text { lectora }\end{array}$ & 2010 \\
\hline $\begin{array}{l}\text { Sánchez-Romero y } \\
\text { Hernández-Prados }\end{array}$ & $\begin{array}{l}\text { Orientaciones didácticas para } \\
\text { la mejora de la comprensión } \\
\text { lectora }\end{array}$ & 2011 \\
\hline Rodríguez-Valls & $\begin{array}{l}\text { Los procesos de calidad en la } \\
\text { enseñanza de la lectura, ori- } \\
\text { gen de un saber democrático y } \\
\text { participativo }\end{array}$ & 2010 \\
\hline Pocoví y Hoyos & $\begin{array}{l}\text { Corriente de desplazamiento: } \\
\text { su presentación en textos y su } \\
\text { comprensión por parte de los } \\
\text { estudiantes }\end{array}$ & 2011 \\
\hline Cassany & $\begin{array}{l}\text { Leer, comprender e interpretar } \\
\text { en EFE en línea }\end{array}$ & 2009 \\
\hline
\end{tabular}

Fuente: elaboración propia.

Según Sánchez y Hernández, “[...] una lectura sin comprensión es una lectura vacía, sin sentido" 
(14). Más que ayudar a leer se debe ayudar a comprender y “[...] centrarse en los exámenes como pieza clave de la educación excluye a menudo la posibilidad de construir un entorno que genere respuestas para el adolescente" (Rodríguez 6). Como se mencionó, es necesaria la búsqueda de nuevas herramientas y estrategias de aprendizaje que permitan resolver interrogantes y llegar a una lucidez en el tema. En este sentido, "[...] los sistemas lingüísticos debieran ayudar al lector en su interpretación de los sistemas simbólicos" (Pocoví y Hoyos 9).

El aspecto lingüístico es un elemento importante para la reconstrucción de significados y, a la vez, puede intervenir en las representaciones simbólicas que se leen en diferentes textos icónicos para obtener una mejor comprensión y análisis de situaciones convencionales o no convencionales.

Chile: en la tabla 4, se observa que las investigaciones tratan del concepto de comprensión lectora y de las teorías sobre este.

Solo durante los últimos años se ha despertado el interés por investigar y alcanzar la comprensión de textos en la universidad, pues únicamente hubo investigaciones del 2009 al 2012, a excepción de los últimos años, en los cuales no existieron nuevos trabajos.

Tabla 4. Investigaciones sobre la comprensión de lectura en Chile

\begin{tabular}{|l|l|l|}
\hline \multicolumn{1}{|c|}{ Autor } & \multicolumn{1}{|c|}{ Título } & Año \\
\hline Makuc & $\begin{array}{l}\text { Teorías implícitas sobre com- } \\
\text { prensión textual y la compe- } \\
\text { tencia lectora de estudiantes de } \\
\text { primer año de la Universidad de } \\
\text { Magallanes }\end{array}$ & 2011 \\
\hline Gacitua-Steman & $\begin{array}{l}\text { Comprensión lectora, pen- } \\
\text { samiento crítico, erudición y } \\
\text { aprendizaje autónomo: concep- } \\
\text { tualización del constructo de } \\
\text { "aprender a aprender" }\end{array}$ & 2012 \\
\hline $\begin{array}{l}\text { Sepúlveda, } \\
\text { Silva-González, } \\
\text { Soto-Ormeño y } \\
\text { Toloza-Salech }\end{array}$ & $\begin{array}{l}\text { Hacia una comprensión interac- } \\
\text { tiva de textos argumentativos }\end{array}$ & 2009 \\
\hline $\begin{array}{l}\text { Maldonado-Fuentes, } \\
\text { Sandoval-Rubilar y } \\
\text { Rodríguez-Alveal }\end{array}$ & $\begin{array}{l}\text { Comprensión lectora en la } \\
\text { formación inicial docente: estu- } \\
\text { diantes de educación general } \\
\text { básica en una universidad del } \\
\text { Consejo de Rectores }\end{array}$ & 2012 \\
\hline Parodi & $\begin{array}{l}\text { La teoría de la comunicabilidad: } \\
\text { notas para una concepción } \\
\text { integral de la comprensión de } \\
\text { textos escritos }\end{array}$ & 2011 \\
\hline
\end{tabular}

Fuente: elaboración propia.
Maldonado, Sandoval y Rodríguez afirman que "[1]a calidad de la educación impulsadas en el país deben apuntar a mejorar el capital cultural de la población estudiantil y con ello los niveles de comprensión lectora desde el ingreso a la educación terciaria" (13). De acuerdo con esto, es importante destacar que en los países se debe implementar un progreso educativo que incluya planes para el mejoramiento cultural y social de los individuos, con el fin de fortalecer los requerimientos que ejerza una persona a nivel de su conocimiento.

Según Makuc, “[...] las teorías implícitas sobre la lectura pueden entregarnos información útil” (15). Esto no solo para que el lector reconozca su perfil lector, sino porque en procesos de intervención cabe explicitar, analizar y profundizar en las teorías implícitas que orientan las decisiones del lector. Esto forma parte integral de las estrategias dirigidas a desarrollar la experiencia lectora en los estudiantes.

Por otra parte, “[...] la comprensión lectora, pensamiento crítico, la erudición temática específica y el aprendizaje autónomo autorregulado pueden en verdad ser integradas bajo un solo constructo conceptual como el de aprender a aprender" (Gacitua 21). Cabe resaltar que "[...] el proceso de lectura-comprensión es una experiencia abierta, no solo personal, que interviene en un espacio también abierto" (Sepúlveda, Silva, Soto y Toloza 23). La razón es que, de acuerdo con lo mencionado, el conocimiento es algo que se construye y para esto es importante compartir nuestros ideales. Además, “[...] los procesos semánticos divergentes que subyacen en textos de diversa naturaleza y que exigen inferencias de tipo diferencial al lector de unos y otros textos de géneros diversos" (Parodi 19).

Otros países: diferentes países tuvieron poca influencia, pero aun así aportan algo a este tema relevante. Se destacan Venezuela, Perú, Cuba, Costa Rica y Uruguay, quienes se exponen en la tabla 5.

La comparación del grupo de países que han realizado investigaciones sobre la comprensión de lectura muestra que casi no se están realizando artículos investigativos que beneficien a una población tan importante como la universitaria. Dicho esto se infiere que estos países latinoamericanos no consideran importante al tema de comprensión de textos en la universidad aun cuando son destacados por el alto nivel educativo de la población. Teniendo en cuenta que muchos adolescentes viajan desde diversos lugares del mundo para culminar sus estudios ahí, se destaca que no solo los jóvenes de colegio y universidad tienen poca formación en la compresión textual, a 
pesar de que sea un asunto de gran importancia, sino que es una cuestión olvidada, sin importar el nivel educativo de que se trate (primaria, secundaria o preparatoria, universidad). Es poca la preocupación y atención que se le ha brindado aun teniendo presente el gran vacío que esto genera en la vida y la experiencia educativa de los estudiantes.

Tabla 5. Investigaciones sobre la comprensión de lectura en Venezuela, Perú, Cuba, Costa rica y Uruguay

\begin{tabular}{|c|c|c|c|}
\hline País & Autores & Título & Año \\
\hline \multirow[t]{2}{*}{ Venezuela } & $\begin{array}{l}\text { Briceño, } \\
\text { Rojas-Velásquez } \\
\text { y Peinado }\end{array}$ & $\begin{array}{l}\text { Influencia de los mapas } \\
\text { conceptuales y los } \\
\text { estilos de aprendizaje } \\
\text { en la comprensión de la } \\
\text { lectura }\end{array}$ & 2011 \\
\hline & $\begin{array}{l}\text { Villamil-Flores, } \\
\text { Arrieta de Meza } \\
\text { y Fuenmayor de } \\
\text { Vílchez. }\end{array}$ & $\begin{array}{l}\text { Análisis de la com- } \\
\text { prensión lectora y } \\
\text { producción escrita de } \\
\text { los estudiantes de educa- } \\
\text { ción media diversificada } \\
\text { y profesional }\end{array}$ & 2009 \\
\hline Perú & $\begin{array}{l}\text { García-Perera y } \\
\text { Monzón-Segura. } \\
\text { Llanos }\end{array}$ & $\begin{array}{l}\text { La comprensión lectora } \\
\text { como pilar esencial } \\
\text { para el aprendizaje del } \\
\text { alumnado en todas las } \\
\text { áreas curriculares } \\
\text { Nivel de compresión } \\
\text { lectora en estudiantes de } \\
\text { primer ciclo de carrera } \\
\text { universitaria }\end{array}$ & $\begin{array}{l}2012 \\
2013\end{array}$ \\
\hline Cuba & $\begin{array}{l}\text { Santiesteban- } \\
\text { Naranjo y } \\
\text { Velásquez-Ávila }\end{array}$ & $\begin{array}{l}\text { La comprensión lectora } \\
\text { desde una concepción } \\
\text { didáctico-cognitiva }\end{array}$ & 2012 \\
\hline Costa Rica & $\begin{array}{l}\text { Solórzano-Salas } \\
\text { y Montero-Rojas }\end{array}$ & $\begin{array}{l}\text { Construcción y valida- } \\
\text { ción de una prueba de } \\
\text { comprensión de lectura } \\
\text { mediante el modelo de } \\
\text { Rasch }\end{array}$ & 2011 \\
\hline
\end{tabular}

Fuente: elaboración propia.

En las investigaciones analizadas se dan a conocer los siguientes aportes: "[...] la comprensión de lectura es una de las habilidades necesarias para el desarrollo pleno de la persona en una sociedad universal, rodeada de múltiples redes de comunicación" (Solórzano y Montero 25). De la misma forma, “[...] los estudiantes carecen de herramientas cognitivas, lo que no les permite lograr el desarrollo de habilidades de comprensión y producción" (Villasmil, Arrieta de Meza y Fuenmayor de Vílchez 2).

De acuerdo con lo planteado, "[...] la comprensión lectora constituye una de las vías principales para la asimilación de la experiencia acumulada por la humanidad" (Santiesteban y Velásquez 2). Resultan de mucho valor algunas herramientas como el "[...] uso de los mapas conceptuales como estrategia, pues, favorece el incremento de los niveles de la comprensión lectora" (Briceño, Rojas y Peinado 14). También depende del modo de leer correctamente: no solo es entonar bien lo que se lee o leer de una manera fluida. "El proceso de la lectura debe ir más allá, puesto que es un proceso de comunicación entre el lector y el texto, sea de la índole que sea" (García y Monzón 1). De esta manera se exponen los parámetros necesarios y adecuados para la comprensión de lectura y, al mismo tiempo, como menciona Osorio, "[...] la comprensión de un texto depende de lo que el lector ya conoce sobre el contenido del texto y del tipo de procesos cognitivos que establezca entre lo que ya se sabe y el contenido de lo que está leyendo" (181).

\section{Ámbito nacional}

Colombia es uno de los países con mayor número de investigaciones sobre la comprensión lectora en universitarios. Se ha conformado una comunidad académica de investigadores al intervenir de manera vigorosa en el tema y ser un objetivo central del Gobierno nacio$\mathrm{nal}^{1}$, y a la vez posee algunos planteamientos que enriquecen el nivel de formación y análisis en cada una de las investigaciones.

En la tabla 6 se muestran las investigaciones relevantes del ámbito nacional sobre la comprensión de lectura en universitarios.

Así mismo, es importante resaltar la participación que ha tenido Colombia en las investigaciones. En estas, hay aportes valiosos dirigidos al mejoramiento de los procesos de comprensión lectora. "Una mejora en la enseñanza y el aprendizaje de la lectura incrementa la puesta en práctica de la democracia de un país" (Haché de Yunén 3). No solo existe un avance educativo, sino también influencia el desarrollo del país, lo que genera opinión sobre los problemas o las dificultades socioculturales de la población. Del mismo modo, "[...] llama la atención la carencia de conceptos de interpretación de la lectura que sean más adecuados, se da dentro del marco de una crisis generalizada de mundo, llamada por algunos teóricos como crisis de civilización" (Álvarez 13). Esta

\footnotetext{
1 En el decreto 80 del 22 de enero de 1980 el presidente de la república de Colombia organizó el sistema de educación postsecundaria y le dio prioridad a la investigación sobre la comprensión lectora según las facultades que le otorga la Ley 8 de 1979. Para mayor información, consúltese el link: http://www.mineducacion. gov.co/1621/articles-102556_archivo_pdf.pdf
} 
falta de conceptos se manifiesta a la hora de interpretar textos; es una falencia que se presenta en muchos países. Los conocimientos previos deben servir para fortalecer y enriquecer estos aspectos que se encuentran con bastante dificultad al momento de realizar una interpretación rigurosa en cada texto.

Londoño indica que "[...] la lectura y la escritura son herramientas sociocognitivas importantes para el desarrollo personal y académico, y representan una opción de cambio sociocultural" (11), pues la lectura y su comprensión van más allá de un estado particular: constituyen a una comunidad en un estado social; es necesario implementar elementos que desarrollen la lectoescritura para beneficio propio y colectivo. Es conveniente resaltar que "[...] los estudiantes, cuando ingresan a la educación superior, se enfrentan a nuevas formas de pensar, indagar, adquirir conocimientos, comprender, interpretar e interactuar con los textos" (Gordillo y Flórez 2). En otras palabras, la lectura en el nivel superior permite que haya ideologías, costumbres, gustos, que nacen de cada lector al adquirir conocimiento a través de su experiencia y del contenido de los textos. En este sentido, se empieza a establecer una mirada crítica ante lo que se propone con el deseo de transformar la visión del lector o para fortalecer aquello que ya sabía.

Tabla 6. Investigaciones sobre la comprensión de lectura en Colombia

\begin{tabular}{|l|l|c|}
\hline \multicolumn{1}{|c|}{ Autores } & \multicolumn{1}{|c|}{ Título } & Año \\
\hline $\begin{array}{l}\text { Gordillo-Alfonso y } \\
\text { Flórez }\end{array}$ & $\begin{array}{l}\text { Los niveles de comprensión lectora: } \\
\text { hacia una enunciación investiga- } \\
\text { tiva y reflexiva para mejorar la } \\
\text { comprensión lectora en estudiantes } \\
\text { universitarios }\end{array}$ & 2009 \\
\hline $\begin{array}{l}\text { Calderón-Ibáñez, } \\
\text { Quijano-Peñuela y }\end{array}$ & $\begin{array}{l}\text { Características de comprensión lec- } \\
\text { tora en estudiantes universitarios }\end{array}$ & 2010 \\
\hline Osorio-Vargas & $\begin{array}{l}\text { Desarrollo de competencias para la } \\
\text { lectura compresiva de textos científ- } \\
\text { cos en el ámbito universitario }\end{array}$ & 2010 \\
\hline $\begin{array}{l}\text { Fajardo, Hernández } \\
\text { y González }\end{array}$ & $\begin{array}{l}\text { Acceso léxico y comprensión lectora: } \\
\text { un estudio con jóvenes universitarios }\end{array}$ & 2012 \\
\hline Londoño-Vásquez & $\begin{array}{l}\text { Análisis sociolingüístico de los niveles } \\
\text { de literacidad en jóvenes de la insti- } \\
\text { tución universitaria de envigado }\end{array}$ & 2012 \\
\hline $\begin{array}{l}\text { Aragón-Espinosa y } \\
\text { Caicedo-Tamayo }\end{array}$ & $\begin{array}{l}\text { La enseñanza de estrategias meta- } \\
\text { cognitivas para el mejoramiento de } \\
\text { la comprensión lectora. Estado de la } \\
\text { cuestión }\end{array}$ & 2009 \\
\hline Haché de Yunén & $\begin{array}{l}\text { Formar lectores, formar ciudadanos. } \\
\text { Impacto social del desarrollo de la } \\
\text { comprensión lectora }\end{array}$ & 2009 \\
\hline
\end{tabular}

\begin{tabular}{|l|l|c|}
\hline \multicolumn{1}{|c|}{ Autores } & \multicolumn{1}{|c|}{ Título } & Año \\
\hline Álvarez-Zapata & $\begin{array}{l}\text { El modo de leer como modo de } \\
\text { producción y consumo textual: ideas } \\
\text { fundamentales de una categoría en } \\
\text { construcción }\end{array}$ & 2010 \\
\hline Rincón y Gil & $\begin{array}{l}\text { Las prácticas de lectura y de escri- } \\
\text { tura académicas en la Universidad } \\
\text { del Valle: Tendencias }\end{array}$ & 2010 \\
\hline Aldana de Becerra & $\begin{array}{l}\text { La lectoescritura en pregrado en el } \\
\text { contexto de la formación investi- } \\
\text { gativa }\end{array}$ & 2013 \\
\hline $\begin{array}{l}\text { Hernández-Nar- } \\
\text { váez, y Ramírez- } \\
\text { Bravo }\end{array}$ & $\begin{array}{l}\text { Comprensión de textos expositivos } \\
\text { en la facultad de medicina }\end{array}$ & 2012 \\
\hline $\begin{array}{l}\text { Olave-Arias, } \\
\text { Rojas-García y Cis- } \\
\text { neros-Estupinán }\end{array}$ & $\begin{array}{l}\text { Leer y escribir para no desertar en la } \\
\text { universidad }\end{array}$ & 2013 \\
\hline Trillos-Pacheco & $\begin{array}{l}\text { La lectura hipermedial y su inci- } \\
\text { dencia } \\
\text { en la comprensión lectora en estu- } \\
\text { diantes universitarios }\end{array}$ & 2013 \\
\hline $\begin{array}{l}\text { Rojas-Noriega y } \\
\text { Jiménez-Mahecha }\end{array}$ & $\begin{array}{l}\text { La educación superior desde la } \\
\text { lectura y la escritura }\end{array}$ & 2012 \\
\hline & \multicolumn{1}{|c|}{ la } & \\
\hline
\end{tabular}

Fuente: elaboración propia.

Se destaca que el reconocimiento de palabras influye en la comprensión lectora. Fajardo-Hernández y González afirman "[...] la lectura implica el procesamiento a múltiples niveles para comprender los textos" (7). Básicamente, para que exista un buen avance hay que descubrir paso a paso los mecanismos que desarrollan la comprensión textual de forma eficaz y que vaya más allá del nivel literal que se presenta en los contenidos. Calderón y Quijano afirman que "[...] los lectores tienen que interrelacionar el significado de las palabras, la sintaxis y las estructuras del discurso", entre otras dimensiones del lenguaje (23). Ayudados de estrategias didácticas, estos factores ayudan a los estudiantes a relacionarse con la lectura y a fortalecer su comprensión desde su entorno para transmitir a otros.

Se puede inferir que "[...] la conciencia y regulación metacognitiva se refieren la capacidad que tienen las personas para planificar estrategias y formas de acercamiento a los textos que faciliten su comprensión" (Aragón y Caicedo 2). Es elemental crear herramientas que satisfagan las necesidades y gustos de los estudiantes por la lectura, así como establecer tipos de textos, incentivar títulos atractivos, realizar escritos, tener un diccionario a la mano. Estas son estrategias que proporcionan una buena comprensión. 
Ahora, un paso muy importante en la formación universitaria, sobre todo en la lectoescritura, es que exista un campo interdisciplinario en el que los estudiantes adquieran un pensamiento enriquecedor con una perspectiva crítica por medio de los docentes, además de que obtengan conocimientos investigativos durante todo el proceso educativo. Por esto, como lo menciona Aldana de Becerra:

La formación investigativa, así como la formación en lectoescritura, no debe ser de responsabilidad exclusiva de las áreas transversales que las tienen a cargo, sino de todo el proceso formativo y durante toda la carrera, de manera que tanto la formación investigativa, como la formación en lectoescritura constituyan una estrategia de autoformación y no una serie de fórmulas descontextualizadas de los procesos formativos. (9)

De igual modo, la lectura favorece procesos investigativos al incidir en el conocimiento de diferentes contextos. "La comprensión lectora implica en términos generales, la habilidad del lector para extraer información a partir de un texto" (Hernández y Ramírez 5). Dado esto, se reitera que los conocimientos y aprendizajes de cada persona son diferentes, pues constituyen una experiencia personal, sin olvidar que todo lo que nos es familiar posiblemente haya sido transmitido por otras personas, lo que hace vital los hábitos lectores: "Acostumbrados a lecturas de orden no académico, se les dificulta abordar tipologías expositivas y argumentativas propias del texto científico" (Olave, Rojas y Cisneros 10). Por tanto, es oportuno resaltar la gran importancia de incentivar la lectura de textos de diferentes tipos desde la etapa infantil.

Es necesario mencionar que en la lectura hipermedial “[...] los jóvenes nativos digitales, al tener más de una ventana abierta, al ingresar a sus cuentas de correo electrónico, al tener más de un texto disponible, al chatear y al utilizar las redes sociales entre otras actividades del ámbito hipermedial" (Trillos 45). Estos jóvenes pueden abrir su mente a un mundo de posibilidades lectoras, lo que puede verse como una ventaja o una desventaja dependiendo del punto de vista, ya que, a pesar de que se cuenta con el acceso casi infinito a una diversidad de textos, esto puede generar la inclinación de los jóvenes por un tipo de escritos, excluyendo los literarios y científicos. Estos últimos son mayormente empleados en las aulas universitarias, es decir, puede llegar a ser una distracción para ciertos fines que pueden ser de más importancia. "Aunque la lectura y la escritura parezca practicas connaturales a las actividades de la vida universitaria, ellas deben ser objeto de problematización y acción reflexiva" (Rojas y Jiménez 14).

\section{Temáticas novedosas y relevantes}

A continuación, se mencionan las temáticas que no fueron abordadas en un principio, pero que luego se consideraron de gran valor con respecto al tema tratado en el artículo. Se tiene en cuenta la participación de la lectura hipermedial y la incidencia que tienen estas estrategias en la comprensión y aprendizaje de los contenidos, con el propósito de implementar ayudas para la comprensión de textos.

Otro tema fundamental es la deserción universitaria, la cual puede verse en diferentes tipos de población sin tener en cuenta edades ni posición socioeconómica. En la deserción influyen diferentes dificultades, desde las estrategias empleadas dentro del aula, los profesores, las instituciones y la parte individual en lo que se refiere a los problemas personales.

\section{Temas no mencionados}

Cabe destacar los temas que no se han tenido en cuenta. En este sentido, no se dan a conocer los mecanismos que se pueden implementar en estudiantes con necesidades educativas especiales. Es evidente que la poca preocupación afecta a los estudiantes en general, al punto de que no se presentan estrategias para el desarrollo de la comprensión textual de estos.

Otro asunto no mencionado es la importancia del uso de las TIC para desarrollar didáctica y pedagógicamente las diferentes destrezas de los estudiantes para que la comprensión de textos resulte mucho más dinámica y activa.

\section{Temas relevantes}

Para esto se establecieron tres temas fundamentales que determinan cuáles son las investigaciones relevantes y significativas que fueron analizadas con anterioridad. Esto se hace con el propósito de conocer cuáles son los aportes que enriquecen la comprensión lectora, como se señala en la tabla 7. 
Tabla 7. Temas relevantes de las investigaciones sobre comprensión lectora en estudiantes universitarios

\begin{tabular}{|l|l|l|}
\hline $\begin{array}{l}\text { Uso y conocimiento de } \\
\text { palabras }\end{array}$ & $\begin{array}{l}\text { Metacognición y } \\
\text { estrategias de com- } \\
\text { prensión lectora }\end{array}$ & $\begin{array}{l}\text { En el contexto } \\
\text { social y cultural }\end{array}$ \\
\hline $\begin{array}{l}\text { Fajardo Hoyos, Hernán- } \\
\text { dez Jaramillo y González } \\
\text { Sierra (Colombia) }\end{array}$ & $\begin{array}{l}\text { Aragón Espinosa } \\
\text { y Caicedo Tamayo } \\
\text { (Colombia) }\end{array}$ & $\begin{array}{l}\text { Haché de Yunén } \\
\text { (Colombia) }\end{array}$ \\
\hline $\begin{array}{l}\text { Morales, Cruz, Silva, } \\
\text { Arroyo y Carpio } \\
\text { (México) }\end{array}$ & Casanny (España) & $\begin{array}{l}\text { Dorronzoro } \\
\text { (Argentina) }\end{array}$ \\
\hline
\end{tabular}

Fuente: elaboración propia.

En la tabla 7, se observan los asuntos relevantes que se mencionaron en los artículos consultados, en los que se destacan definiciones, comprensión, enseñanza de estrategias y formar en la práctica; estos temas han sido abarcados por la mayoría de los países en los últimos años.

\section{Referentes teóricos}

A continuación se mencionarán los referentes teóricos que dieron cabida a las investigaciones analizadas. Se resaltarán algunos de estos por sus destacados aportes y por su mayor credibilidad en este importante trabajo, tales como, Trillos-Pacheco, que sugiere el uso competente de una serie de estrategias para promover nuevos aprendizajes.

Otros referentes son Carrasco, que señala la diferencia entre aprender a leer y leer, pues la escuela enseña a leer, a reconocer un sistema de representaciones escritas, pero esto no a comprender, y Casanny, que sugiere, desde una perspectiva estrictamente lingüística o psicológica, que se entiende la lectura como una actividad básicamente verbal y cognitiva. Estos autores destacan la importancia, las estrategias, aspectos y factores que se encuentran en la comprensión de lectura partiendo de todas las inferencias.

\section{Metodología}

El $60 \%$ de las investigaciones adopta un enfoque cualitativo y el $31 \%$, uno cuantitativo. También se tiene un enfoque cualitativo al indagar y analizar los aportes de autores que precedente las investigaciones consultadas, como lo demuestran Saniesteban y Velásquez, Makuc, y Giraldo y Villa.

En las investigaciones con un enfoque cualitativo, se observaron detalladamente sus datos, las cuestiones formuladas, las informaciones estadísticas y de análisis. Los textos revisados son los de Calderón y Quijano, Briceño, Rojas y Peinado, y Gordillo y Flórez.

Por último, el 9\% de las investigaciones adoptan los dos enfoques, se relacionan entre sí, analizando parte por parte los aportes y la recolección de datos como encuestas, entrevistas análisis y aportes teóricos como Sánchez e Ibañez y Quijano.

\section{Resultados}

Algunos resultados de las investigaciones consultadas y que aportan al tema de la comprensión lectora en los estudiantes universitarios son:

Las teorías implícitas sobre la lectura pueden entregar información útil, no solo para que el lector reconozca su perfil lector, sino que en procesos de intervención cabe explicitar, analizar y profundizar en las teorías implícitas que orientan las decisiones del lector como parte integral de las estrategias dirigidas a desarrollar la experticia lectora en los estudiantes. (Makuc 15)

Si leer está asociado con la comprensión y aprendemos más fácil lo que comprendemos, esto hace imperativo desarrollar métodos y estrategias que se adapten a las exigencias de una educación que satisfaga las necesidades del estudiante y la sociedad. (Monroy y Gómez 5)

\section{Discusión}

Los resultados del estado del arte contribuyen a comprender los factores que influyen en los procesos de comprensión lectora de estudiantes universitarios, y se atiende a la pregunta: ¿cuál es el estado del arte de las investigaciones sobre comprensión de lectura en estudiantes universitarios?

La investigación mostró las dificultades en la comprensión lectora de estudiantes universitarios. Por consiguiente, los estudiantes deben poner de su parte al leer los textos implicados en la carrera y nutrir su conocimiento por iniciativa propia, además de ser guiado por un profesor en el aula durante el proceso de lectura.

Este estudio documental reveló que los estudiantes universitarios presentan problemas en todos los niveles señalados por el modelo de comprensión lectora utilizado en la investigación; pero, especialmente, en la representación mental de textos científicos.

La verdad del caso es que muchas veces no se enseña lectura y escritura en las diferentes áreas de la 
carrera universitaria. Los profesores no contemplan dentro de su labor enseñar a leer y a escribir debido a que, según ellos, no es tan esencial y es algo que compete a los maestros de la escuela. Por el contrario, presuponen que ocuparse de la lectoescritura es impropio de la universidad.

Es importante capacitar a los docentes universitarios sobre cómo diseñar herramientas y aplicarlas para que sus estudiantes puedan acceder al conocimiento que los docentes dominan, pero dentro de una interdisciplinariedad.

\section{Conclusiones}

Durante los últimos años, en los países hispanos, se ha tratado muy poco la comprensión de lectura en los trabajos investigativos. Por motivos diversos los profesores no se preocupan por el nivel de comprensión e interpretación que tienen los universitarios sobre textos científicos.

No obstante, algunos países, como México y Colombia, se han preocupado por abordar este problema por medio de investigaciones en que se han podido observar la tendencia en los textos actuales que son leídos por los universitarios, la importancia e índices de comprensión lectora. Lastimosamente, muchos países optan por poner la investigación en el contexto implícito del diálogo, lo que incrementa las limitaciones de esta. Esto lleva a involucrarse en un tema delicado que deja de lado los puntos fuertes del trabajo y sus implicaciones en el campo disciplinar.

Por último, son muy poco frecuentes las aplicaciones de estrategias ante la incomprensión de textos de universitarias debido a que muchas veces hasta los profesores son escépticos con respecto a esto.

\section{Referencias}

Aguilar-Trejo, José Luis, Alberto Ramírez-Martinell y Rocío López-González. "Literacidad digital académica de los estudiantes universitarios: un estudio de caso". Revista Electrónica de Investigación y Docencia (2014). Web. 24 de marzo del 2015. <http://revistaselectronicas.ujaen. es/index.php/reid/article/viewFile/1257/1083>.

Aldana de Becerra, Gloria Marlén. "La lectoescritura en pregrado en el contexto de la formación investigativa”. Revista Virtual Universidad Católica del Norte 39 (2013). Web. 25 de febrero del 2015. <http://www.redalyc.org/ articulo.oa?id=194227509008>.
Alfaro-López, Héctor Guillermo. (2010). "La lectura como proceso de comprensión y conocimiento científico". Investigación Bibliotecológica 24.50 (2010): 35-47. Web. 25 de febrero del 2015: <http://www.ejournal.unam. $\mathrm{mx} / \mathrm{ibi} / \mathrm{vol} 24-50 / \mathrm{IBI002405003.pdf>}$.

Álvarez-Zapata, Didier. Del modo de leer como modo de producción y consumo textual: ideas fundamentales de una categoría en construcción. Revista Educación y Pedagogía 14.32 (2010): 137-147. Web. 24 de marzo del 2015. <http://aprendeenlinea.udea.edu.co/revistas/index.php/revistaeyp/article/view/6739/6172>.

Aragón-Espinosa, Lucero y Adriana María Caicedo-Tamayo. "La enseñanza de estrategias metacognitivas para el mejoramiento de la comprensión lectora. Estado de la cuestión". Pensamiento Psicológico 5.12 (2009): 125 138. Web. 25 de febrero del 2015. <http://portales. puj.edu.co/psicorevista/components/com_joomlib/ ebooks/PS12-9.pdf>.

Briceño, Luis Alberto, Freddy Rojas-Velásquez y Sofía Peinado. "Influencia de los mapas conceptuales y los estilos de aprendizaje en la comprensión de la lectura". Revista Estilos de Aprendizaje 8.8 (2011): 1-20. Web. 25 de febrero del 2015. <http://www.uned.es/revistaestilosdeaprendizaje/numero_8/articulos/lsr_8_articulo_1.pdf>.

Bustos-Pérez, Eva María. "Dificultades de la comprensión lectora”. Revista digital Innovación y Experiencias Educativas 37 (2010). Web. 25 de febrero del 2015. <http:// www.csi-csif.es/andalucia/modules/mod_ense/revista/pdf/Numero_37/EVA_BUSTOS_2.pdf>

Calderón-Ibáñez, Arlenys y Jorge Quijano-Peñuela. “Características de comprensión lectora en estudiantes universitarios". Revista Estudios Sociojurídicos 12.1 (2010): 337-364. Web. 25 de febrero del 2015. <http://revistas.urosario.edu.co/index.php/sociojuridicos/article/ view/1195>.

Cartoceti, Romina, Valeria Abusamra, Bárbara Sampedro y Aldo Ferreres. "Influencia del mecanismo de actualización en la comprensión de textos". La renovación de la palabra en el bicentenario de la Argentina. Los colores de la mirada lingüística. Ed. Víctor M. Castel y Liliana Cubo de Severino. Mendoza: Facultad de Filosofía y Letras Universidad de Cuyo, 2010. Impreso.

Cassany, Daniel. (2009). Leer, comprender e interpretar en EFE en línea. 2009. Ts. Universidad Pompeu Fabra. Web. 25 de febrero del 2015. <http://cvc.cervantes.es/ ensenanza/biblioteca_ele/asele/pdf/20/20_0019.pdf $>$.

Castro-Azuara, María Cristina y Mario Sánchez-Camargo. "La expresión de opinión en textos académicos 
escritos por estudiantes universitarios". Revista Mexicana de Investigación Educativa 18.57 (2013). Web. 24 de marzo del 2015. <http://www.redalyc.org/ pdf/140/14025774008.pdf>.

Cepeda-Islas, María Luisa. María López-Gamiño y Carlos Santoyo-Velasco. "Relación entre la paráfrasis y el análisis de textos". Revista Electrónica de Investigación Educativa 15.1 (2013). Web. 24 de marzo del 2015. <http:// www.scielo.org.mx/scielo.php?pid=S1607-40412013 000100008\&script $=$ sci_arttext $>$.

Demagistri, María Silvina y Liliana María Naveira. "Comprensión de textos científicos en adolescentes". La renovación de la palabra en el bicentenario de la Argentina: Los colores de la mirada lingüística. Ed. Víctor M. Castel y Liliana Cubo de Severino. Mendoza: Facultad de Filosofía y Letras Universidad de Cuyo, 2010.

Dorronzoro, María Ignacia. "Las prácticas de lectura y escritura en el contexto de la educación superior. Una aproximación sociocultural". Polifonías, Revista del Departamento de Educación II.3 (2013): 85-100.

Fajardo, Adriana, Janeth Hernández y Ángela González. "Acceso léxico y comprensión lectora: un estudio con jóvenes universitarios". Revista Electrónica de Investigación Educativa 14.2 (2012): 25-33. Web. 25 de febrero del 2015. <http://redie.uabc.mx/redie/article/ view/305/660>.

Fernández de Castro, Javier. "Perspectivas en torno al desarrollo de la comprensión lectora". Revista de Evaluación Educativa 2.1 (2013). Web. 25 de febrero del 2015. <http://revalue.mx/revista/index.php/ revalue/article/viewArticle/65>.

Gacitua-Steman, Andrés. "Comprensión lectora, pensamiento crítico, erudición y aprendizaje autónomo: conceptualización del constructo de 'aprender a aprender". Revista Electrónica de Desarrollo de Competencias 5.1 (2012): Web. 25 de febrero del 2015. <http:// redec.utalca.cl/index.php/redec/article/view/130>.

García-Perera, Gustavo y Jesús Monzón-Segura. "La comprensión lectora como pilar esencial para el aprendizaje del alumnado en todas las áreas curriculares" Revista de la Carrera de Lenguaje y Literatura 1 (2012). Web. 24 de marzo del 2015. <http:// www3.gobiernodecanarias.org/medusa/ecoescuela/ tamadaba/2012/01/11/la-comprension-lectora-como-pilar-esencial-para-el-aprendizaje-del-alumnado-en-todas-las-areas-curriculares/>.

González-Hernández, Klency. (2009). “Diseño de un programa para mejorar la comprensión de textos en estudiantes universitarios". Revista Latinoamericana de Estudios
Educativos 39.1 (2009): 125-151. Web. 25 de febrero del 2015. <http://www.redalyc.org/pdf/270/27015065006. pdf $>$.

Gordillo-Alfonso, Adriana y María del Pilar Flórez. "Los niveles de comprensión lectora: hacia una enunciación investigativa y reflexiva para mejorar la comprensión lectora en estudiantes universitarios". Revista Actualidades Pedagógicas 53 (2009): 95-107 Web. 25 de febrero del 2015. <http://revistas.lasalle.edu.co/index.php/ ap/article/viewFile/1048/953>.

Guerra-García, Jorge y Yolanda Guevara-Benítez. "Validación de un instrumento para medir comprensión lectora en alumnos universitarios mexicanos”. Enseñanza e Investigación en Psicología 18.2 (2013): 277-29 Web. 25 de febrero del 2015. <http://www.cneip.org/documentos/revista/CNEIP_18_2/277.pdf>

Guevara-Benítez, Yolanda y Jorge Guerra-García. "La comprensión lectora como competencia genérica”. Revista Electrónica de Psicología Izcatala 16.2 (2013). Web. 25 de febrero del 2015. <http://www.revistas.unam.mx/ index.php/repi/article/view/39950>.

Haché de Yunén, Ana Margarita. "Formar lectores, formar ciudadanos. Impacto social del desarrollo de la comprensión lectora”. Revista Tendencias \& Retos 14 (2009): 135-143. Web. 24 de marzo del 2015. <http://revistas. lasalle.edu.co/index.php/te/article/view/1287/1176>.

Heit, Ignacio. "Estrategias metacognitivas de comprensión lectora y eficacia en la asignatura Lengua y Literatura”. Revista de Psicología 8.15 (2012). Web. 28 de febrero del 2015. <http://bibliotecadigital.uca.edu.ar/repositorio/revistas/estrategias-metacognitivas-comprension-lectora.pdf $>$.

Hernández-Narváez, Edith y Roberto Ramírez-Bravo. “Comprensión de textos expositivos en la facultad de medicina”. Revista EDUCY (2012):1-14. Web. 24 de marzo del 2015. <http://revistas.udenar.edu.co/index.php/duniversitaria/article/view/1441/0>.

Hernández-Suárez, Irais y Bernardo Riffo. "Desarrollo de la competencia lectora de textos científicos en tres etapas de formación universitaria”. Revista LiminaR: Estudios Sociales y Humanísticos. 7.2 (2009). Web. 24 de marzo del 2015. <http://www.redalyc.org/articulo. oa? id $=74516319008>$.

Llanos-Cuentas Olimpia. "Nivel de compresión lectora en estudiantes de primer ciclo de carrera universitaria". Tesis de maestría en Educación con mención en Teorías y Práctica Educativa. Universidad de Piura, 2013. Web. 5 de octubre del 2015. <http://pirhua.udep. 
edu.pe/bitstream/handle/123456789/1814/MAE_ EDUC_106.pdf?sequence $=1>$.

Londoño-Vásquez, David Alberto. (2012). "Análisis sociolingüístico de los niveles de literacidad en jóvenes de la institución universitaria de envigado". Tesis de maestría. Universidad de Manizales y Cinde. Web. 24 de marzo del 2015. <http://ridum.umanizales.edu. co:8080/jspui/handle/6789/310>.

Makuc, Margarita. “Teorías implícitas sobre comprensión textual y la competencia lectora de estudiantes de primer año de la Universidad de Magallanes". Estudios Pedagógicos 32.1 (2011): 237-254. Web. 25 de febrero del 2015. <http://mingaonline.uach.cl/pdf/estped/v37n1/ art13.pdf $>$.

Maldonado-Fuentes, Ana Carolina, Pedro Sandoval-Rubilar y Francisco Rodríguez-Alveal. "Comprensión lectora en la formación inicial docente: estudiantes de educación general básica en una universidad del Consejo de Rectores". Folios 35 (2012): 33-47. Web. 28 de febrero del 2015. <http://www.scielo.org.co/pdf/folios/n35/ n35a03.pdf>.

Mendes Pessoa Pullin, Elsa, Natália Moraes-Góes, y Talita Kito. "El interés de los futuros profesores en leer textos de estudio". Rmie 18.59 (2013). Web. 24 de marzo del 2015. <http://www.redalyc.org/articulo. oa?id=14028945010>.

Monroy-Romero, José Alberto y Blanca Estela GomézLópez. "Comprensión Lectora". Revista Mexicana de Orientación Educativa. 6.16 (2009): 37-42. Web. 25 de febrero del 2015. <http://pepsic.bvsalud.org/pdf/ remo/v6n16/v6n16a08.pdf>.

Moore-Hanna, Pauline y Esther Narciso Sánchez. "Modelos epistémicos de la lectura en estudiantes universitarios mexicanos". Revista Mexicana de Investigación Educativa. 16.51 (2011): 1197-1225. Web. 3 de marzo del 2015. $<$ http://www.redalyc.org/articulo.oa?id=14019203009>.

Morales, Germán, Nadia Cruz, Alejandro León, Héctor Silva, Rosalinda Arroyo y Claudio Carpio. "Morfología y función en el análisis empírico del ajuste lector". Suma Psicológica, 17.1 (2010). Web. 24 de marzo del 2015. $<$ http://publicaciones.konradlorenz.edu.co/index. php/sumapsi/article/view/168/387>.

Olave-Arias, Giovanny, Ilene Rojas-García y Mireya Cisneros-Estupiñán. "Leer y escribir para no desertar en la universidad". Folios 38 (2013): 45-59. Web. 24 de marzo del 2015. <http://www.scielo.org.co/pdf/folios/n38/ n38a04.pdf $>$.

Osorio-Vargas, Elsa. (2010). "Desarrollo de competencias para la lectura compresiva de textos científi- cos en el ámbito universitario". Tesis Universidad de Manizales. Web. 24 de marzo del 2015. <http:// bibliotecavirtual.clacso.org.ar/Colombia/alianza-cinde-umz/20110412112008/tesis-osorio-v.pdf>.

Parodi, Giovanni. "La teoría de la comunicabilidad: notas para una concepción integral de la comprensión de textos escritos". Revista Signos 44.76 (2011). Web. 3 de marzo del 2015. <http://www.scielo.cl/scielo.php?pi$\mathrm{d}=$ S0718-09342011000200004\&script=sci_arttext $>$.

Pocoví, María Cecilia y Elena Hoyos. "Corriente de desplazamiento: su presentación en textos y su comprensión por parte de los estudiantes". Enseñanza de las Ciencias 29.2 (2011): 275-287. Web. 8 de marzo del 2015. <http://ddd.uab.cat/pub/edlc/edlc_ a2011v29n2/02124521v29n2p275.pdf>.

Rincón, Gloria y John Gil. "Las prácticas de lectura y de escritura académicas en la Universidad del Valle: tendencias". Escuela de Ciencias del Lenguaje 38.2 (2010). Web. 8 de marzo del 2015. <http://daca.univalle.edu. co/politica_curricular/Practicas-de-lectura-y-de-escrituraacademicas-en-la-univalle-tendencias.pdf>

Rodríguez-Valls, Fernando. "Los procesos de calidad en la enseñanza de la lectura, origen de un saber democrático y participativo". Revista Iberoamericana sobre Calidad, Eficacia y Cambio en Educación 8.5 (2010): 119-132. Web. 28 de febrero del 2015. <http://www. redalyc.org/articulo.oa?id=55119084009>.

Rojas-Noriega, Guillermina y Hermínsul Jiménez-Mahecha. "La educación superior desde la lectura y la escritura". Revista Amazonia Investiga 1.1 (2013): 19-35. Web. 3 de marzo del 2015. <http://www.udla.edu.co/revistas/ index.php/amazonia-investiga/article/view/3>.

Salas-Navarro, Patricia. El desarrollo de la comprensión lectora en los estudiantes del tercer semestre del nivel mediosuperior de la universidad autónoma de nuevo león. Web. 5 de octubre del 2015. <http://eprints.uanl. $\mathrm{mx} / 3230 / 1 / 1080256466 . p d f>$.

Sánchez-Romero, Elisa Isabel y María Ángeles Hernández-Prados. "Orientaciones didácticas para la mejora de la comprensión lectora”. I Congreso de Internacional Virtual de Educación Lectora. 7 de marzo del 2011. Web. 3 de marzo del 2015. <http://puertotics. wikispaces.com/file/view/sanchezh2.pdf/211281750/ sanchezh2.pdf>.

Santiesteban-Naranjo, Ernan y Kenia Velásquez Ávila. "La comprensión lectora desde una concepción didáctico-cognitiva”.Didasc@lia: Didáctica y Educación 3.1 (2012): 103-110. Web. 25 de febrero del 2015. <http:// www.hacienda.go.cr/cifh/sidovih/uploads/archivos/ 
Articulo/La\%20comprensi\%C3\%B3n\%20lectora\%20 desde $\% 20$ una $\% 20$ concepci\%C3\%B3n\%20did\%C3\%A1ctico-cognitiva-2012.pdf>.

Saux, Gastón, Débora Burin, Natalia Irrazabal y Carlos Molinari-Marotto. "Inferencias causales durante la comprensión de textos expositivos en formato multimedia”. Perspectivas en Psicología 9 (2012) Web. 5 de octubre del 2015. <http://www.seadpsi.com.ar/revistas/index.php/pep/article/view/113/59>.

Sepúlveda, Miguel, Karen Silva-González, Pablo SotoOrmeño y Alejandra Toloza-Salech. "Hacia una comprensión interactiva de textos argumentativos". Revista de Estudios y Experiencias en Educación 8.16 (2009): 65-84. Web. 25 de febrero del 2015. <http:// www.redalyc.org/articulo.oa?id=243116384005>.

Solórzano-Salas, Julieta y Eliana Montero-Rojas. "Construcción y validación de una prueba de comprensión de lectura mediante el modelo de Rasch". Actualidades Investigativas en Educación 11.2 (2011): 1-27. Web. 20 de febrero del 2015. <http://revista.inie.ucr.ac.cr/ index.php/aie/article/view/455/454>.
Trillos-Pacheco, Juan José. "La lectura hipermedial y su incidencia en la comprensión lectora en estudiantes universitarios". Palabra Clave 16.3 (2013): 944-992. Web. 3 de marzo del 2015. <http://palabraclave.unisabana. edu.co/index.php/palabraclave/article/view/2664>.

Vélez, Carlos Mario. Apuntes de metodología de la investigación. Medellín: Universidad Eafit, 2001. Web. 5 de octubre del 2015. <http://guerrero.upn.mx/chilpancingo/images/stories/METODOLOGIA_DE_LA_INVESTIGACION.pdf $>$.

Vega-López, Norma Alicia, Gerardo Bañales-Faz y Antonio Reyna-Valladares. "La comprensión de múltiples documentos en la universidad". Revista Mexicana de Investigación Educativa 18.57 (2013). Web. 24 de marzo del 2015. <http://www.redalyc.org/pdf/140/14025774007. pdf $>$.

Villasmil-Flores, Yeriling, Beatriz Arrieta de Meza y Gloria Fuenmayor de Vílchez. "Análisis de la comprensión lectora y producción escrita de los estudiantes de educación media diversificada y profesional". Multiciencias 9.1 (2009): 62-69. Web. 3 de marzo del 2015 $<$ http://132.248.9.34/hevila/Multiciencias/2009/vol9/ no1/8.pdf $>$. 